\title{
Developing QoS by Priority Routing for Real Time Data in Internet of Things (IoT) Urban Scenarios
}

\author{
Radwan S. Abujassar \\ Information Technology and Computing (ITC) \\ Arab Open University, Kuwait Branch \\ r.abujassar@aou.edu.kw
}

\begin{abstract}
In networks, many application protocols such as CoAP, REST, XMPP ,AMQP have been proposed for IoT communication which includes p2p or S2S. In MANET Network convergence does the way for improvements in Internet of Things (IoT) communication with high potential for a wide range of applications. Each protocol focuses on some aspects of communication in the IoT. Hence, these application protocols have indicated of how IoT has integrated to enhanced and developed of a new service that require to guarantees the wide range offered by the quality of services. In this paper, we will introduce a smart pathway that can be bridge the gap between IoT services with its real data traffic. Therefore, we enhanced the MANET routing protocol for computing two or more paths to pass the more that one high priority real traffic data via these paths to improve the gloomy picture of this protocol in the context of IoT. In particular, the good services with high timely delivery of urgent data such as real time data environmental monitoring. After surveying the published and available protocol interoperability given for urban sensing. In this research, we have proposed a novel solution to integrate MANET overlays, and collaboratively formed over MANET, to boost urban data in IoT. Overlays are used to dynamic differentiate and fasten the delivery of high priority real application time data over low-latency MANET paths by integrating with the original specifications. Our experimental results showed the effectiveness on the network such as the overhead and network congestion. In addition, the initial results of the light-weight improved the routing protocol over the baseline protocols in terms of the delay of reciveing the packets between nodes which lead to increase the throughput by reducing loss packets.
\end{abstract}

Keywords-component; Quality of Service (QoS),Adhoc on demand Distance Vector Alternative (ODVA), Open Link State Routing (OLSR), Internet OfThing(IOT)

\section{Introduction}

The Internet protocol (IP) is defined as a connectionless datagram protocol which is used to forward data packets from one host to another within a network. Each host has an IP address, therefore the location for each host can be defined according to its address [1] [2]. In IP, the data packets are divided into chunks that are sent separately to the destination. There are two types of IP addresses: IP version 4 (IPv4) and IP version 6 (IPv6) . The former is used in networks for $99.9 \%$ of addresses $[3,4]$. The packets are forwarded from the source to its destination hop-by-hop, using a set of hardware called "router", which is implemented to send the data packets to the final end-host by checking the IP destination address, which is found in the IP packet header.The work presented in paper showed that good improvements have been made in network performance by finding an alternative path for moving data traffic to run the required application. While many published

DOI: $10.5121 /$ ijcnc.2019.11603 
research solutions moved to reduce the impact of many existing techniques on the network performance. We provided a new research studies based upon node density's in MANET network with varying speed movements from slowly to fast mobility in the network topology. The main idea of this paper is to improve and develop the network performance while data packets passing faster between nodes in the networks. However, the challenge in the MANET network is that the flexibility of node movements results have produced more overhead and delay during route maintenance. Flooding of control packets can form a harmful problem due to the sharing of wireless media, upon which the loss of packets is high and difficult to avoid [5]. In the proposed scenario, we have Increased the number of nodes inside the terrain area which will lead to gain better results by taking less time to find an alternative pathway. Hence, the high density leads to retaining connections for longer times than low densities. When the MANET network contains low density nodes, then when the nodes keep moving frequently that will lead to form an instantaneous disconnected graph between source and destination, then packets that need to be received will be dropped or lost. This work will support video traffic delivery across a different number of nodes in a network topology. The novelty of this paper is to optimise number of adacent nodes in the network topology, and provide a high reliable networks by creating more than one alternative paths between source and destination node. In this paper, the overall proposed solution (alternative path) was to evaluate the ability of AODV routing protocol to react for network topology changing while packets keep sending over source to destination by trying to deliver them successfully. The evaluation is based on the simulation with different number of nodes forming a network, moving and changing their positions over a rectangular terrain area. The extension code was implemented and configured in AODV protocol to compute a different path during the convergence of the network topology.

The rest of the paper is organized as follows. In section 2, we discuss related works about incentive schemes including previous studies for the IoT and it's relation of improving QoS in the network. In section 3, we proposed our techniques and the mechanisms as well as some mathematical theory for the configurations and theoretical analysis for the proposed technique. The results of our experiments have been evaluated and discussed is in section 4.2. Finally, a conclusion and future work is discussed in section 5.

\section{Related Works}

In wireless communications and mobile devices are starting new services through novel integration opportunities. It is widely recognized that MANETs network is coming to be the key technologies for several IoT application domains in smart cities that are using internet widely[5]. In addition, many researchers show that many municipalities will soon adopt MANETs, mainly for public safety, localization, and environmental monitoring [6]. we proposed opportunistic exploitation of MANETs to produce a very high speed up forwarding data while MANET nodes (relays) in a smart city. However, many approaches relay are neither mobile harvesters that convey sensed data to the Internet, nor mobile WSN users only. Table-1 discuss and showed their most important features: as each protocol is 
targeted at specific niches and, as a consequence, has a various balance in the trade-off between range distance between all surrounding node [6].

\begin{tabular}{|l|l|l|l|l|l|l|l|}
\hline Name & $\begin{array}{l}\text { Standard } \\
\text { body }\end{array}$ & $\begin{array}{l}\text { Standard } \\
\text { document }\end{array}$ & $\begin{array}{l}\text { Physical } \\
\text { specification }\end{array}$ & $\begin{array}{l}\text { Nominal } \\
\text { Range } \\
\text { (OdBm) }\end{array}$ & $\begin{array}{l}\text { Data } \\
\text { rate }\end{array}$ & $\begin{array}{l}\text { Network } \\
\text { specification }\end{array}$ & IPv6 support \\
\hline Bluetooth LE & $\begin{array}{l}\text { Bluetooth } \\
\text { SIG }\end{array}$ & $\begin{array}{l}\text { Bluetooth } \\
\text { Core 4.0 }\end{array}$ & $\begin{array}{l}\text { Yes } \\
\text { (short range, high } \\
\text { data rate) }\end{array}$ & $10 \mathrm{~m}$ & $1 \mathrm{Mbps}$ & Star topology & $\begin{array}{l}\text { Yes (IETF } \\
\text { draft) }\end{array}$ \\
\hline DASH7 & $\begin{array}{l}\text { DASH7 } \\
\text { Group, } \\
\text { ISO }\end{array}$ & $\begin{array}{l}\text { ISO/IEC } \\
18000- \\
7: 2009\end{array}$ & $\begin{array}{l}\text { Yes } \\
\text { (long range, low } \\
\text { data rate) }\end{array}$ & $250 \mathrm{~m}$ & $\begin{array}{l}28-200 \\
\text { Kbps }\end{array}$ & No & No \\
\hline RPL & IETF & $\begin{array}{l}\text { IETF ROLL } \\
\text { WG }\end{array}$ & No & N/A & N/A & Mesh & Yes \\
\hline ZigBee & $\begin{array}{l}\text { ZigBee } \\
\text { Alliance, } \\
\text { IEEE }\end{array}$ & $\begin{array}{l}\text { IEEE } \\
802.15 .4- \\
2006\end{array}$ & $\begin{array}{l}\text { Yes } \\
\text { (medium range, } \\
\text { medium data rate) }\end{array}$ & $75 \mathrm{~m}$ & $\begin{array}{l}250 \\
\text { Kbps }\end{array}$ & Mesh & Yes (draft) \\
\hline
\end{tabular}

Fig. 1. Table-1: Standard Protocol and Main features

\subsection{MANET and Ad Hoc Routing Protocol Proposal}

In this section, we showed some on-demands protocol with explaining the most important cluster information by detailing packet exchanges in the MANET layer .In MANET protocol merging MANET clusters by classified the nodes in which willbe formed in localized areas that need urgently packets to be exchanged the transmission between source and destination. In our technique, we explored that a two-hops limit are good trade-off between routing protocol improvements and cluster robustness. As we have confirmed by our experimental test results. In addition, as we consider the number of hop also the distance range between nodes can lead to form different path between each cluster which will make the data transfer more faster as there is an alternative path. The cluster formation protocol is reactively started by each node in the MANET network, called cluster group and one one node will be nominated as a cluster head, that snoops an urgent data packet being routed on the MANET to target node. As in Fig. 4, the protocol consists of three phases. In the first phase, each network will be divided into cluster and each cluster will select a head node then extracts from the sniffed packet on the gradient network. In the second round test, the MANET network node's are received the join new one a request by sending a checking small packets to all nodes in the same range. They show the it with the gradient declared in the join request: only the MANET nodes can communicate with each node in the cluster network. In the third and final round, MANET nodes communicate to the cluster-head node that they joined the new cluster and get confirmed then update the routing table. The cluster-head collect responses from all nodes and then selects an exit point. Collecting answers from all the nodes joined in its cluster's, to allow node head to estimate the number of messages that MANET and mobile will exchange: appropriate policies can determine how long he integration should last, giving guarantees on its path requirements for the aim node [13] [7]. 


\section{IoT architecture}

The idea behind IoT was started at the Massachusetts Institute of Technology (MIT) from a work at the Auto-id center in 1999. The MIT has conducted many researches in networked radio identification (RFID) and emerging sensing Technologies (Wikipedia: The free encyclopedia, November, 2014). Recently, million devices or more were connected online while the population was estimated to be more increased [8]. The ratio of devices per person was almost one person to two devices smart phone,tablet and smart watch. Today, with the increase in technological innovation and the continuous growth of smart phones. In a research conducted in China, the authors showed that the internet doubles its size every 5.32 years. With this result it is showing that the number of nodes will be communiacte with themselves to get a secure and safty communication among the adjacent nodes [9].

Currently, Many research have been published on the IoT as curently cinsider very importnat topicc and widely start to be used in smart and modern cities. The IoTis coming to propose a three-tier layers structure which it can be defined by its functionlity by containing of a perception layer, network layer and application layer [10]. This is further explained below:

- Perception Layer : is the sense organ of IoT. It aims at recognizing objects and collecting information. This layer includes RFID tags, 2-D barcode labels and readers, terminals, GPS, camera, sensors and sensor network.

- The network layer :This layer represents the confluence of IoT. It processes and sending information received from the perception layer to the application layer. The network layer comprises of the following:information center, intelligent processing center, Internet network systems and network management center.

- The Application Layer : This layer represents the confluence of IoT and industrial technology with a mix of industrial needs and machine intelligence[11].

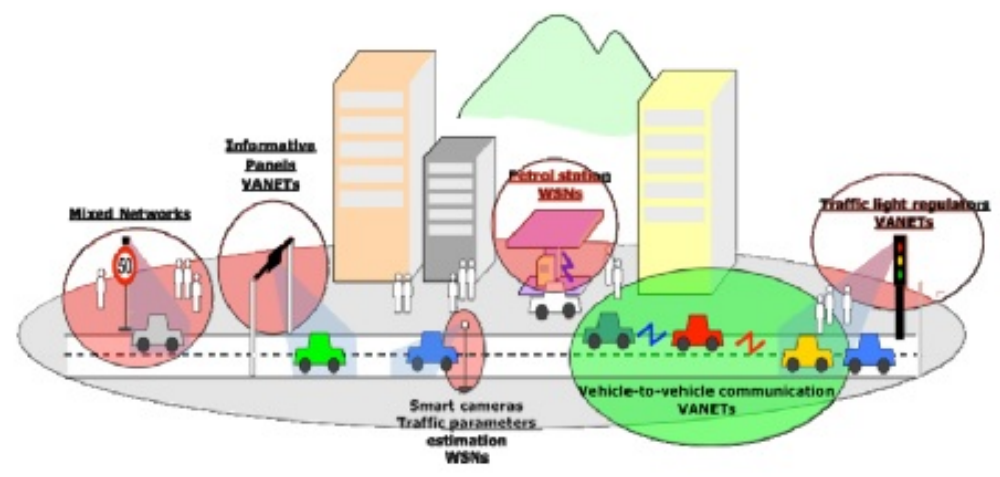

Fig. 2. Example of different networks in IOT 
In figure 2, we represent a new paradigm of communications where decentralized wireless nodes communicate with each other in a collaborative way to achieve a common goal for the transmission data packets. The Nodes in the figure show how they can collaborate to establish unicast or multicast communications between a source node and a one or several destination node(s). When mobility of odes is considered, communications refer to Mobile Ad Hoc Networks (MANETs). With the increment of mobile devices which are equipped with wireless transceivers such as smart phones, tablets, sensors and so on, the number of employed devices with wireless communications capabilities is continuously increasing. When vehicles are capable of exchanging information among them Vehicular Ad Hoc Networks (VANETs) are formed [12][13].

\subsection{MANETS Routing Protocol}

Different routing protocols have been published for different environments to improve network performance when connectivity is lost by nodes moving or failing [14-17]. In [18], the authors mentioned some of MANET's characters and environments, such as, Bandwidth (BW), resources. Some proactive routing protocols (such as Destination-Sequenced Distance-Vector Routing (DSDV), Optimized Link State Routing Protocol (OLSR), the Cluster head Gateway keep changing or switching Routing Protocol (CGSR) and Wireless Routing Protocol (WRP)) keep tracking the messages that can detect links connection problem when they go down $[19,20]$. Based on these packets, the routing protocol can build and maintain paths to its destination. In re-active protocols, such as, DSR, AODV (On-demand Distance Vector) they faced a high overheads because of huge LS packets forwarding while the routing table is constructed so it will be usability for main pathway between source and destination. In [21,22], it is showing that the OLSR had Multi Point Relay (MPR) nodes to pass link state packets to construct a routing table for the network topology. In OLSR protocol, each node will broadcast a Hello packets to its adjacent every $2 \mathrm{sec}$ to determine that if the connectivity is on or getting down as a waiting period of relying is $6 \mathrm{sec}$ as it is considered too long time. The interval time is five seconds. The waiting time is $15 \mathrm{sec}$ to permit and confirm failure has been occurred. After OLSR routing protocol converge the MANET network then all nodes will begin transmitting packets. Depending on interval times for the Hello packets and the time has been needed for updating routing table with topology information, the backup path will be used to reroute the data via the new path; this will lead to increased loss of data packets and reduced throughput [24]. With respect to node density, in [25], 60 nodes were created and distributed in a $1200 \mathrm{~m} \times 800 \mathrm{~m}$ terrain area [26].

\subsection{Features of MANET Networks}

A MANET is an independent topology of mobile nodes connected by wireless radio connections. These nodes are free to move in a random form; therefore, the topology of wireless networks can updated swiftly and in an unpredictable pattern. Generally, direct com- 
munication in MANET is possible only between adjacent nodes, and communication between distant nodes is established upon multiple-hops based on the routing table, which is computed by a routing protocol. The locations of these nodes may change dynamically; hence, the interconnections between the adjacent nodes may change frequently, therefore the seeking of an efficient routing protocol is required to adopt these changes and update network information faster, whilst trying to reduce the number of control messages in the network. Each node can be form as a host or router, relaying data traffic from one node to another. In Figure 3 shows a small ad hoc network containing three hosts or nodes. As the figure shows, not all nodes are in the same range, so the node in the middle acts as an intermediate node that can be used to forward data packets between the two other nodes.

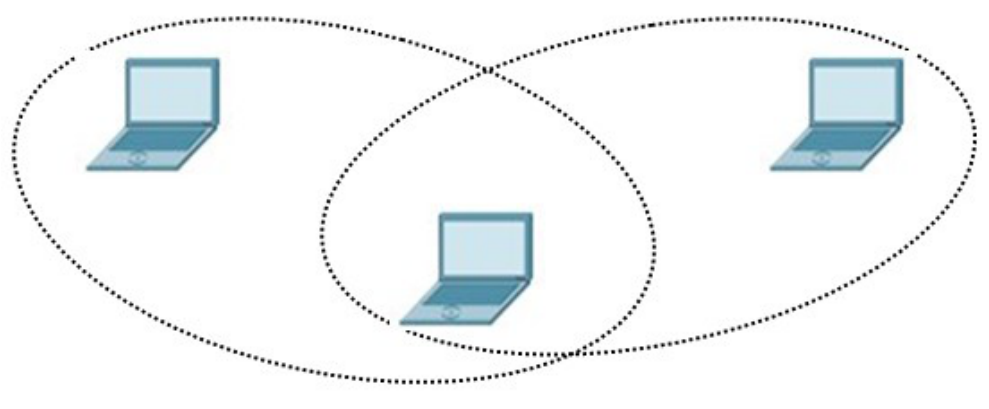

Fig. 3. Wireless Node

Characterisation of Node Stability In Mobile Ad-hoc networks, each node has a physical location due to flexible node movement in the network. However, slow or fast mobility of the mobile nodes impacts upon the network stability. In neighbourhoods, the frequent change of the topology by joining or departing from the existing node, leads to increased control message overhead, to update the routing table for the proactive protocol, such as link state or distance vector. Because of the limiting power of nodes in ad hoc network, the transmission range may also be limited [27].There are two concepts models in ad hoc network widely used: free space and Ground Reflection two-way models. The free space is an ideal propagation model because it uses a basic reference model. The transmission power can be expected through this modelling, when there is un-obstructed line of sight between sender and receiver. The free space model uses the equation below for non-isotropic antennas [28]:

$$
P_{r}=P_{t}\left(\frac{\lambda}{\pi 4 d}\right)^{2} G_{t} G_{r}
$$

Where $\mathrm{P}_{r}$ is the received energy, $\mathrm{P}_{t}$ is the sender power (in Wts or mili-Wts), $\lambda$ is the carrier wavelength (in meter), $\mathrm{d}$ is the distance between sender and receiver (in meters); 
$\mathrm{G}_{t}$ is the antenna get at the sender and $\mathrm{G}_{r}$ is the antenna get at the receiver. Where antenna gain is known as the power output for a determine direction. However, the equation shown as below:

$$
P_{d b}=10 \log _{10}\left(\frac{P_{\text {out }}}{P_{\text {in }}}\right)
$$

For the ideal omni-directional antenna, the free space loss equation is:

$$
\frac{P_{t}}{P_{r}}=\frac{(4 \pi d)^{2}}{(\lambda)^{2}}=\frac{(4 \pi f d)^{2}}{c^{2}}
$$

The following equation is used to calculate the receiver power and radio range distance for two-ray model [28]:

$$
P_{r}=P_{t} G_{t} G_{r}\left(\frac{h_{t}^{2} h_{r}^{2}}{d^{4}}\right)
$$

Where $\mathrm{G}_{t}$ is the transmitter antenna gain; $\mathrm{G}_{r}$ is the receiver for the topology; $\mathrm{d}$ is the current distance between the antennas in meters; $h_{t}$ is the height of the transmitter antenna; $\mathrm{h}_{r}$ is the height of the receiver antenna.

\subsection{Classified of Routing Protocol in MANET's}

Based on the terrain area and distance between nodes, several hops may be needed to reach the destination node. Hence, a routing protocol comes with two main functions: to find the best route between source node and its destination, ensuring that the data packets are delivered at the right destination; and to construct the routing table for the network topology to build a full view for each node in the network topology.

\section{Design Guidelines and Protocol Overview with IoT Services}

Figure 4 explain how proposed technique works between form the required path with IoT services. Each node on the MANET network will connect with more than one adjacent node and between each node there is hop and next-hop. because we use the clustering according to the path rout between nodes and checking the adjacent node according to the distance in MANET network then there are many nodes surrounding with also called adjacent node. According to the configured on-demand routing protocol, one rout can be made for exchanged the data traffic between source and destination. However, Each router has been selected to be on the main path will be able to buffer data with its adjacent to pass the priority data traffic. We perform different number of topologies, which acts a good example to discuss our proposed technique. In this topology, each node has at least two neighbours that can use its adjacent for tolerate the exchange packets through either one of them when it is needed. There are many paths between $\mathrm{S} \rightarrow \mathrm{D}$ as follow:

$\mathrm{S} \rightarrow 1 \rightarrow 3 \ldots \rightarrow \mathrm{D}, \mathrm{S} \rightarrow 1 \rightarrow 4 \rightarrow \ldots . \mathrm{D}, \mathrm{S} \rightarrow 1 \rightarrow 5 \rightarrow 7 \rightarrow \ldots \rightarrow \mathrm{D}$ The total weights for all the topology $\mathrm{W} 0=\mathrm{X} ; \mathrm{X}$ : Total weights for all $\operatorname{arcs}$ on the topology. Then:W=W0=X; 


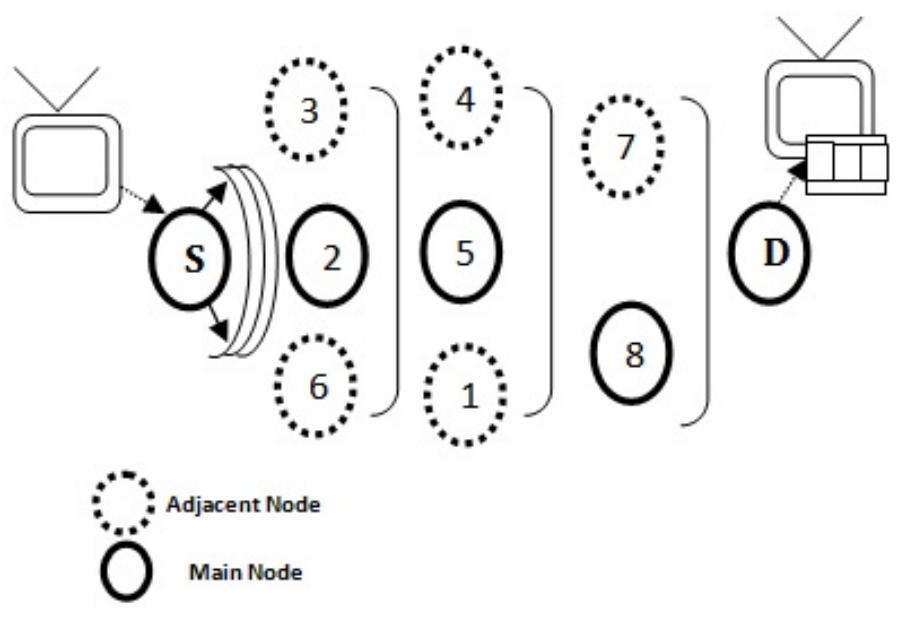

Fig. 4. :MANET Topology Node

If we assume the shortest path between $\mathrm{S} \rightarrow \mathrm{D}$ after the dijkstra algorithm has computed it as follow: $\mathrm{Ps} \rightarrow \mathrm{DS} \rightarrow 1 \rightarrow 5 \rightarrow 7 \rightarrow \ldots . . \rightarrow \mathrm{D}$ Hence, our new technique will add the total cost to the primary path that will lead to exclude all edges on the primary path to be chosen in the backup path as follow: Step 1: in case fig.2.1, the main shortest pathway $\mathrm{Ps} \rightarrow \mathrm{D} \mathrm{S} \rightarrow$ $1 \rightarrow 5 \rightarrow 7 \rightarrow \ldots \rightarrow \mathrm{D}$ is computed, and it is labelled as the first agreed path.

Step 2: Every edge on $\mathrm{P}$ has its weight increased by $\mathrm{W}=\mathrm{W} \_0=\mathrm{X}+\mathrm{W} \_0$. Then, we re-invoke the its adjacent for using its path with same range for buffering and passing the priority traffic from node which is connected directly. Ps $\rightarrow \mathrm{D} \mathrm{S} \rightarrow 1 \rightarrow 5 \rightarrow 8 \rightarrow \ldots \rightarrow \mathrm{D}$ We illustrates when $\mathrm{S}$ node broadcasting for label their adjacent in different locations then all nodes on the primary path can use same mechanism. This is will increase the QoS such as reduce delay and increase the throughput $\mathrm{Ps} \rightarrow \mathrm{DS} \rightarrow 1 \rightarrow 3 \rightarrow \ldots \rightarrow \mathrm{D}$ In this Example, we illustrate how the adjacent node constructs an additional next-hops for the destination. Node S: Construct candidate routing For $S$ into the destination Route via si 1 or 2 Node 1: Construct candidate hop for neighbour- 1 to the destination. Route via $s \rightarrow 1 \rightarrow 3$ or 4 or $5 \rightarrow \mathrm{D}$ depend on the cost and which is non on the primary path Node 2: Construct candidate next-hop for neighbour- 2 to the destination. Route vias $\rightarrow 5$ or $6 \rightarrow$ D Node 3 : Construct candidate next hop for neighbour- 2 to the destination. Route via $\mathrm{s} \rightarrow 1 \rightarrow 3 \rightarrow \mathrm{N}$ or $\mathrm{Y} \rightarrow \mathrm{D}$

In this defention-1, when the source sends packets via any next-hop nodes towards the destination, the packet for no reason returns to the source. Three conditions are proposed for loop freeness in the network:

(1) $\mathrm{C} 1(\mathrm{v} ; \mathrm{d})<\mathrm{C} 1(\mathrm{~s} ; \mathrm{d})$

(2) $\mathrm{Cj}(\mathrm{s} ; \mathrm{d})+\mathrm{c}(\mathrm{s} ; \mathrm{NHj}(\mathrm{s} ; \mathrm{d}))<\mathrm{C} 1(\mathrm{~s} ; \mathrm{d})$

(3) $\mathrm{Cj}(\mathrm{s} ; \mathrm{d})=\mathrm{C} 1(\mathrm{~s} ; \mathrm{d})$ In condition-1, the source inquiry its adjacent node for best route better than its own pathway. Condition- 2 the source will find an alternate pathway with 
loop free between nodes on the topology to avoid overhead. On the other hand, condition3 its for Equal Cost Multiple Path network topology.

In network communications, using second paths between source and destination provide a solution for networks when there are data traffic with high priority need to be exchanged. The algorithm was evaluating the node distance with explore an alternative pathway from the existing main path which is already in the routing table to ensure that the new pathway will use next-hop not in the main one. Hence, algorithm 4 will calculated the distance between nodes to detect all adjacent within radio propagation range $250 \mathrm{~m}$. If the distance between two nodes is less than $250 \mathrm{~m}$, then the proposed algorithm will work between all nodes with their adjacent. When there is more than one adjacent node, then it starts to test which adjacent node has a free new path to the destination. However, the computation algorithm evaluates an appropriate next node for configure the route as it is references from the main one which it is tracking from source to destination. The nodes linked to the main path will be not involved from the new alternative backup pathway. Each node will take place in a coordination position (X, Y) randomly under the radio propagation range for the network area. In algorithm 2, the nodes on the MANET topology are broadcasting a small control messages to enquire from adjacent nodes if they have available and reliable backup path from its primary path.

Algorithm 1 ComputePrimaryRoutingTable returns a set of less weight pathway for each node on the network

Algorithm 1 executes the following steps to construct the routing table:

- Set small Distance $=\infty$

- Compute Propagation Radio Range as follow:

$$
P_{r}=P_{t} G_{t} G_{r}\left(\frac{h_{t}^{2} h_{r}^{2}}{d^{4}}\right)
$$

Where $\mathrm{G}_{t}$ is the transmitter antenna gain; $\mathrm{G}_{r}$ is the receiver antenna gain; $d$ is the distance between the antennas in meters; $\mathrm{h}_{t}$ is the height of the transmitter antenna; $\mathrm{h}_{r}$ is the height of the receiver antenna. Configure transmitting power $\mathrm{P}_{t}$ is 0.28 with range distance $250 \mathrm{~m}$.

- Calculate the distance between hosts

- X, Y the coordinate position for the nodes in network model which are allocated randomly.

$$
d=\sqrt{\left(x_{2}-x_{1}\right)^{2}+\left(y_{2}-y_{1}\right)^{2}}
$$

- if (Dist < 250) insert node to the list of adjacent.

- Compare the adjacent nodes which one has the smallest distance to be the first next hop in the primary routing table.

- Calculate the pathway $\mathrm{P}$ with the less number of hops to the destination.

We assume that each node can be on the primary path and it can determine its adjacent node as an alternative next hop after the on-demand routing protocol starts working. As shown below, there are some of the rules that must be achieved with its demanded. We 
addressed the following rules in all experiments we have test our technique as follows: The adjacent router should not be affected by joining any other mobile node . We have illustrated the proposed algorithm in 2.The adjacent node must have a different next hop as an egress point which form a path into the destination. The algorithm has proved that it's efficiency through the theoretical analysis and it's implementation on the NS2 simulator. The analysis has shown how the alternative next-hop will be labelled to be activated when it is needed.

\subsection{Theoretical Analysis for the Proposed Algorithm}

In on-demand routing protocol, the packets are sending to the destination via an acceptable quality metrics. On other words, the pre-active protocol maintain up the routing table up to date and periodically. We have considered a graph, $\mathrm{G}=(\mathrm{V}, \mathrm{E})$, where $\mathrm{V}$ is the set of nodes, and $\mathrm{E}$ is the set of edges. A virtual edge exists between the nodes according to the node in the same range. We assume in the proposed algorithm that the each node can be labelled it is next-hop in MANET network as pointing in $T_{r}$. Then the nodes can know her adjacency's through the routing table as $\Gamma(v)$. Hence we will define the path from source to destination as follow:

$p_{a}(s, d) \leftarrow \emptyset$

Theorem: For On-demand protocol, the next hop can be find within the each node range hop by hop until the full path will be fully computed. By using the adjacency matrix, the run time is $\mathrm{O}\left(n^{2}\right)$ and if we used an adjacency linked list; it is a partially ordered tree as a data structure for organizing of the set $\{\mathrm{V}-\mathrm{S}\}$.

-Assume there is a $\mathrm{G}(\mathrm{V}, \mathrm{E}), \mathrm{V}$ the number of nodes; $\mathrm{E}$ number of edges between nodes. The design of network should contain different number of nodes $\{n\}$ The number of edges $=2^{n}$. This is because it can offer multinext hop for each node.

Let: $\mathrm{V}=1,2,3 \ldots \ldots, \mathrm{n}-1$, and assume the source node is $\{0\}$; Cost of arc $(\mathrm{i}, \mathrm{j})$ initially $=\infty$ Initially: The set $\mathrm{V}=1,2,3 \ldots \mathrm{n}-1$ which contain all nodes; $\mathrm{S}=0$ to push all nodes have the best path. Source Node is $\{0\}$ and $\mathrm{K}=\emptyset$; which is the set of alternative edges to the destination when the primary path goes down. Choose a vertex $\mathrm{w} \in \mathrm{V}-\mathrm{S}$, such that $\mathrm{D}[\mathrm{w}]$ which is a minimum distance; $\mathrm{S}=\mathrm{S} \cup \mathrm{w}$; For each vertex $\mathrm{v} \in \mathrm{V}$-S

$\mathrm{D}[\mathrm{v}]=\min (\mathrm{D}[\mathrm{v}], \mathrm{D}[\mathrm{w}]+\mathrm{C}[\mathrm{w}, \mathrm{v}]) \mathrm{S}=0$, all nodes with next-hop path until arrived the final destination

If the primary path need to pass data traffic with high priority, then the computations will be as follow:

$$
\mathrm{S}=\mathrm{S} \cup\{\mathrm{w}\} \div\{\mathrm{K}\} ; \text { For each vertex } \mathrm{v} \in \mathrm{V}-\{\mathrm{S} \& \mathrm{~K}\}
$$

$\mathrm{D}[\mathrm{v}]=\min (\mathrm{D}[\mathrm{v}], \mathrm{D}[\mathrm{w}]+\mathrm{C}[\mathrm{w}, \mathrm{v}])$

Therefore,we defined a random variable $\mathrm{X}_{j} \in\{0,1\}$ indicates to the link propagation status between any two routers $\mathrm{A}$ and $\mathrm{B}$ of a sub region. The index of $\mathrm{j}$ indicates to the time when A forwards the update packets to the node head. The sequence of $X_{0}, 1, \ldots . ., X_{j} . .=\left\{X_{j}\right\}_{j}$ $\infty=1$. The random processes $\left\{\mathrm{X}_{j}\right\}_{j} \infty$ is modelled as a Markov chains. Here,the proposed new algorithm has shown the aims of the function through computed a next-hop which 
can be the optimum next hop one by one until getting the full path between source and destination. In section 4.2, we have proved that the proposed algorithm has achieved some enhancements on the receiving traffic between source and destination.

\subsection{Simulation environment}

A network simulation (NS2) was performed to evaluate the performance of the proposed alternative next hop based on the on-demand protocol between nodes in the MANET networks. A comparison of the simulation results of the OLSR,DSDV as a pro-active protocol with AODV and Ad hoc On-Demand Distance Vector Alternative (AODVA) protocol. The evidence gathered by the NS2 simulation offered good support for the transmission data in the networks. At the network link layer, the researcher used the IEEE 802 Ethernet. While the simulation is running, every node tests its adjacent node to begin building its backup pathway.The simulation was running more than ten times and an average among all results were calculated. The packet size was $512 \mathrm{~KB}$ and the bit rate was set to $2 \mathrm{Mb} / \mathrm{s}$. A traffic rate of $200 \mathrm{~kb} / \mathrm{s}$ was generated from the source node to the destination during the simulation.

Each topology shows a comparison between the OLSR,DSDV, AODV and AODVA routing protocols operating with and without calculating an alternative path for calculate the required one while changing the number of nodes in the topology frequently. Before evaluating the performance of network topologies, and with respect to calculating next-hop over network's topology, it is an important to consider what are network parameters could be affect the QoS of real time traffic. Hence, the research concentrate on three parameters, which may improved reveal the affect of real time traffic:

- Packet loss ratio: the packet ratio between loss and forwarding data packets.

- Average e2e delay: the average time between sending and receiving data packets.

However, we have make an extension on the AODVA protocol by performing our algorithm 2 as below for preparing an adjacent to be next hop if it faced the required conditions. As we discussed before, we add enquiry packets with negligible sized to find the next-hop as an alternative for urgent packets.

In order to check the effection node's density, it is very critical to know the number of nodes in each topology. This is because, number of nodes densities enables to discover and maintain hop by hop according to the node numbers. When the number of nodes increases then more alternative hops will become more available. When the number of nodes is decreased then the number of alternative adjacent will be diminish; however, the computing of alternate hop will be completed in very short time based on speed of movements. In addition, big number of nodes lead to an produce more in the probability of path breaking compared to when the number of nodes is reduced. We also computed the delay for different numbers of nodes with different types of network topology.The main parameters of the simulation are configured based on the table as below 1 . 


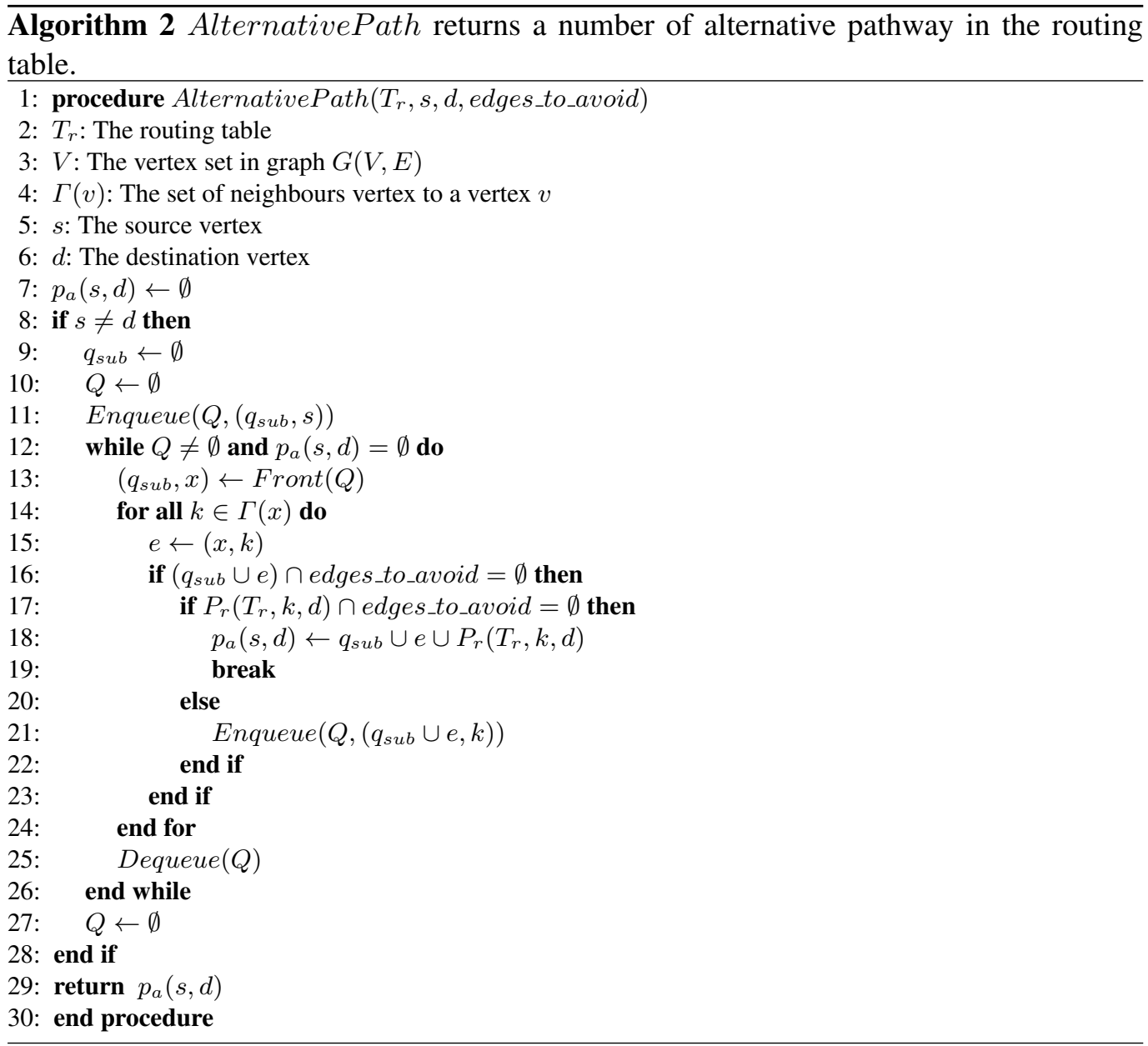

\begin{tabular}{|l|l|}
\hline Parameter & Value \\
\hline Wireless technology & IEEE 802.11 \\
\hline Max. range & $250 \mathrm{~m}$ \\
\hline Roaming area & $1000 \times 800 \mathrm{~m}^{2}$ \\
\hline No. of Nodes & 25 \\
\hline Pause Time & $10,30,60,90 \mathrm{~s}$ \\
\hline Min. Speed & $0 \mathrm{~m} / \mathrm{s}$ \\
\hline Max. Speed & $10 \mathrm{~m} / \mathrm{s}$ \\
\hline Mobility model & Random WayPoint \\
\hline Routing protocol & $\begin{array}{l}\text { OLSR AODV, DSDV, } \\
\text { AODV-IOT,OLSR-BK }\end{array}$ \\
\hline
\end{tabular}

Table 1. Simulation Parameter for Backup Path with Pausing Time 
In figure 5, we have monitored the effect of variety nodes in each cluster topology on packet ratio delivery. it is known that increasing the node density by one, the service duration distributions follow the same pattern. In reality, routing messages marginally increased in order to update the information for each node on the topology about its path. This means that very improved scaling properties were found when the density raised. The size of LS messages has a very important role in high-density topology where congestion and overhead are occurred. When number of nodes are high or topology contain high number of nodes , the packet loss will be increased; This is because high number of the inquiry packets which increased the congestion. Meanwhile, the availability of finding alternative path will be very high. Hence, the loss of packets will increase in relation to the frequent changes in the node informations. In this case, the nodes around the source can pass the packets directly between them, which reduce the waiting time for the packets inside the nodes buffers.

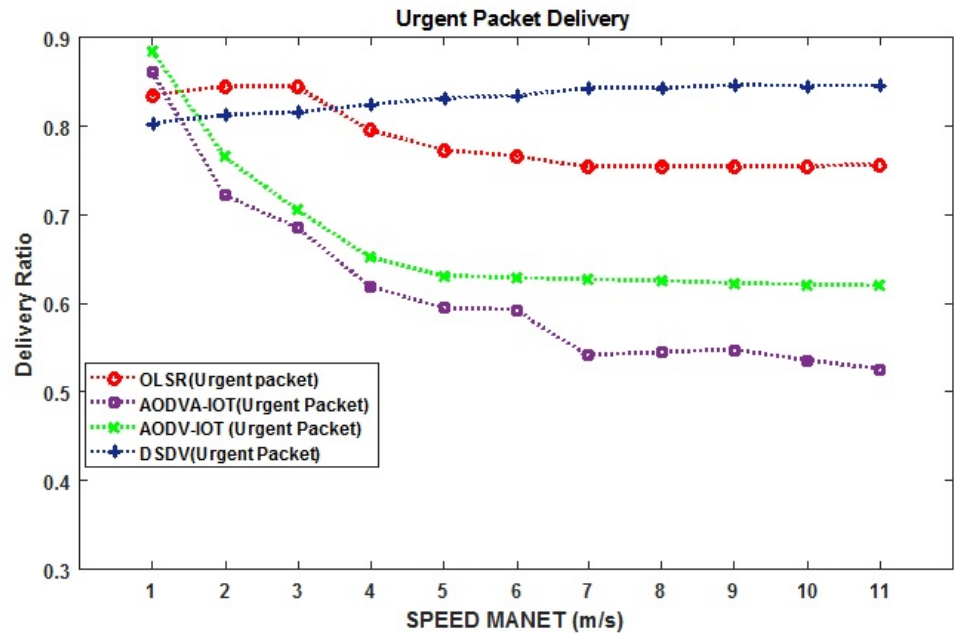

Fig. 5. Deliver Urgent Packet Latency from transmitter to receiver

As we expected, the proposed solution reduced delivery latency of urgent and high priority packets in every tested topology. More interestingly, the produced results identify the optimise number of nodes in the MANET that grants the best latency improvements. With more than 30 nodes in the topology, latency slightly getting high due to the additional data packets induced by advertising and discovery packets, which lead to raise more data packet collisions. Regarding to the speed nodes, if the number of nodes increased then still the fast response for the urgent packet still high regarding to the number of an adjacent node.

On the other hand, when the destination node is getting more further from the source node, then each node will try to keep its data packets in its buffer if there is any space until it 


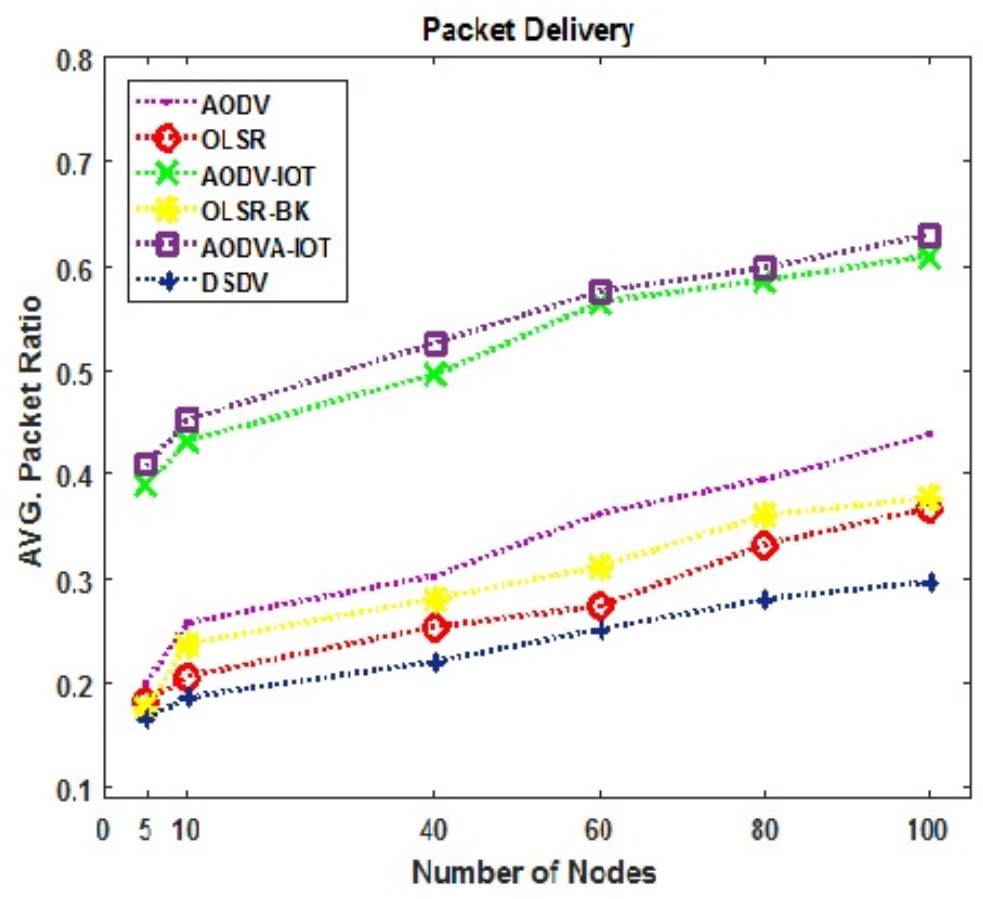

Fig. 6. Deliver Packet from Source to Destination

can find an available adjacent next hop based on the proposed technique. If a node receives data that exceed the size of the buffer then it will start to drop them. so , our algorithm is directly converted the data packets via alternative hop to get the destination without dropping. In minimum node density in the topology, the loss of packets will be reduced but somehow with conditions; we mean here the movements speed for the node should be very slow. In addition, finding and calculating an alternative pathway between source and destination will be faster than in topology has a high node density. In figure 6 , we have proved that the data packets delivery improved comparing with on-demand and pro-active protocol. The proposed AODVA protocol has shown a bit better improvement comparing with AODV-IOT one. this is because the algorithm try to consider the best adjacent based on an algorithm 4 to determine that this adjacent node will be an alternative hop.

In figure 7 shows the destination node received the packet from the source node in less time compared with other protocols with respect to the two ways that have been used for choosing the next hop mechanism. We can see that the AODVA protocol produces a linear line graph because the number of adjacent node increased. In addition, the failure detection and calculating times are very small for constructing alternative pathway. However, AODVA also showed good results by redirected the data packets in less time; this is because some nodes moving suddenly out of its range but it casn detect the loss of connection in less time. 


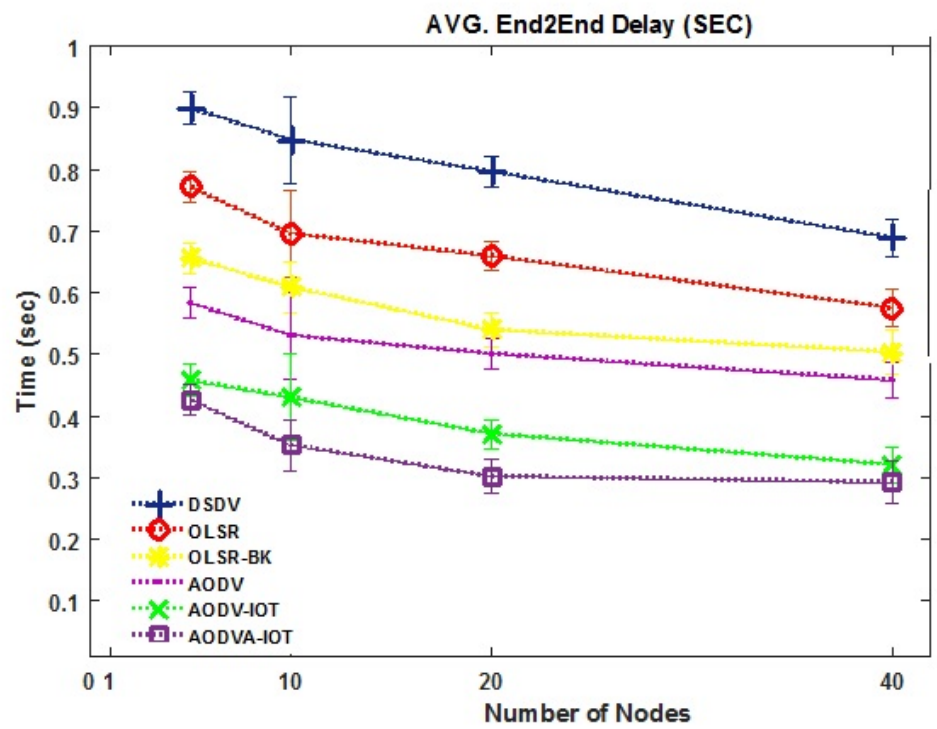

Fig. 7. Deliver Packet from Source to Destination

In high density topology, comfortable showing is experienced for real time application traffic. The continuity index is getting less when the number of nodes become high. Bearing in mind the time of the receiving packets spend in the buffer and in updating next hop. However, according to the graph, we show a high continuity index for the urgent traffic in relation to congestion in the network.

\section{Conclusion}

IoT based services and applications are already becoming an important part of our everyday life. New technologies that leverage seamless interaction between lower and upper layer in the network. These interactive lead to equipped Internet of things and impromptu MANET networks opportunistically formed by humans moving in the smart city. Hence, the raised number of using mobility node will play a relevant role in different application domains, such as logistics and traffic management. We believe that our proposed algorithm represents a step forward toward the development of novel real-world deployment solutions for the real time traffic. The proposed protocol algorithm's will be capable to help the full potential MANET network in the IoT in order to support the fast collection of high density nodes topology according to the number of users. The algorithm showed some improvements on the real time traffic transmission in IoT for smart cities: ubiquitous and collaborative urban sensing of keep moving with smart objects. The improvements of receiving real dat time packets can be lead to enhance citizen life by providing an intelligent environment. Once the improvements occurred then the services lead to reduced 
loss of packets. On the one hand, we are investigating the cases of exploiting our solution in other different IoT application, such as in Intelligent cloud computing storage data services to integrate vehicular networks and on-the-road as we considering the mobility between nodes.

\section{ACKNOWLEDGEMENTS}

This study was supported and funded by research sector, Arab Open University -Kuwait Branch under grant number 18091

\section{References}

1. Marzieh Hamzei and Nima Jafari Navimipour. Toward efficient service composition techniques in the internet of things. IEEE Internet of Things Journal, 5(5):3774-3787, 2018.

2. K. Egevang and P. Francis. The ip network address translator (nat). http://www.hjp.at/doc/rfc/rfc1631.html, 1994.

3. F. Baker. Requirements for ip version 4 routers. https://tools.ietf.org/html/rfc1812, 1995.

4. R. Hinden and S. Deering. Ip version 6 addressing architecture. http://tools.ietf.org/html/rfc4291, 2006.

5. Y. Tseng, S. Ni, Y. Chen, and J. Sheu. The broadcast storm problem in a mobile ad hoc network. Wireless networks, 8(2):153-167, 2002.

6. Paolo Bellavista, Giuseppe Cardone, Antonio Corradi, and Luca Foschini. Convergence of manet and wsn in iot urban scenarios. IEEE Sensors Journal, 13(10):3558-3567, 2013.

7. K Pradeep Kumar and BR Prasad Babu. Dsmanet: Defensive strategy of routing using game theory approach for mobile adhoc network. In Computer Science On-line Conference, pages 311-320. Springer, 2018.

8. IA Alameri. Manets and internet of things: the development of a data routing algorithm. Engineering, Technology \& Applied Science Research, 8(1):2604-2608, 2018.

9. Daniel Giusto, Antonio Iera, Giacomo Morabito, and Luigi Atzori. The internet of things: 20th Tyrrhenian workshop on digital communications. Springer Science \& Business Media, 2010.

10. Bandar H Al-Qarni, Ahmad Almogren, and Mohammad Mehedi Hassan. An efficient networking protocol for internet of things to handle multimedia big data. Multimedia Tools and Applications, pages 1-18, 2018.

11. Waheb A Jabbar, Wasan Kadhim Saad, and Mahamod Ismail. Meqsa-olsrv2: A multicriteria-based hybrid multipath protocol for energy-efficient and qos-aware data routing in manet-wsn convergence scenarios of iot. IEEE Access, 6:76546-76572, 2018.

12. Saleem Ulla Shariff, MG Gurubasavanna, and CR Byrareddy. Iot-based smart food storage monitoring and safety system. In International Conference on Computer Networks and Communication Technologies, pages 623-638. Springer, 2019.

13. Amar Amouri, Vishwa T Alaparthy, and Salvatore D Morgera. Cross layer-based intrusion detection based on network behavior for iot. In Wireless and Microwave Technology Conference (WAMICON), 2018 IEEE 19th, pages 1-4. IEEE, 2018.

14. X. Zeng, R. Bagrodia, and M. Gerla. Glomosim: a library for parallel simulation of large-scale wireless networks. In Proceedings. Twelfth Workshop on Parallel and Distributed Simulation, 1998. PADS 98., pages 154-161. IEEE, 1998.

15. R. Dube, C. Rais, K. Wang, and S. Tripathi. Signal stability-based adaptive routing (ssa) for ad hoc mobile networks. In Personal Communications, IEEE, volume 4, pages 36-45. IEEE, 1997.

16. C. Toh. A novel distributed routing protocol to support ad-hoc mobile computing. In Conference on Computers and Communications, 1996., Conference Proceedings of the 1996 IEEE Fifteenth Annual International Phoenix, pages 480-486. IEEE, 1996. 
17. D. Johnson, D. Maltz, and J. Broch. Dsr: The dynamic source routing protocol for multi-hop wireless ad hoc networks. Ad hoc networking, 5:139-172, 2001.

18. D. Kim, J. Garcia, and K. Obraczka. Routing mechanisms for mobile ad hoc networks based on the energy drain rate. IEEE Transactions on Mobile Computing, 2(2):161-173, 2003.

19. D. Park and M. Corson. A highly adaptive distributed routing algorithm for mobile wireless networks. In Proceedings of Conference of the IEEE Computer and Communications Societies. Driving the Information Revolution (INFOCOM) Sixteenth Annual Joint, page 1405. IEEE Computer Society, 1997.

20. J. Moy. Link-state routing in routing in communications networks. http://www.faqs.org/rfcs/rfc2328.html, 1995. M.E. Steenstrup, Prentice Halls.

21. P. Narula, S. Dhurandher, S. Misra, and I. Woungang. Security in mobile ad-hoc networks using soft encryption and trust-based multi-path routing. Computer Communications, 31:760-769, 2008.

22. C. Dhote, M. Pund, R. Mangrulkar, and R. Makarand. Article: Hybrid routing protocol with broadcast reply for mobile ad hoc network. International Journal of Computer Applications, 1(10):108-113, 2010. Published By Foundation of Computer Science.

23. E. Baccelli and J. Antonio. Ospf over multi-hop ad hoc wireless communications. International Journal of Computer Networks \& Communications IJCNC, 2(5):37-56, 2010.

24. J. Broch, D. Maltz, D. Johnson, Y. Hu, and Jetcheva. A performance comparison of multihop wireless ad hoc network routing protocols. In Proceeding of International Conference Mobile Computing and Networking (MobiCom) ACM, pages 85-97, 1998.

25. J. Broch, D. Maltz, D. Johnson, Y. Hu, and J. Jetcheva. A performance comparison of multi-hop wireless ad hoc network routing protocols. In Proceedings of the 4th annual ACM/IEEE international conference on Mobile computing and networking, pages 85-97. ACM, 1998.

26. Sankar Mukherjee and GP Biswas. Networking for iot and applications using existing communication technology. Egyptian Informatics Journal, 19(2):107-127, 2018.

27. R. Zoican. Analysis of scalability in manet's protocols. In Conference on Telecommunications in Modern Satellite, Cable and Broadcasting Services,TELSIKS 8th International, pages 605-608. IEEE, 2007.

28. T. Rappaport. Wireless communications: principles and practice, volume 2. Prentice Hall New Jersey, 1996. 